\title{
Spotting of over Speeding Vehicles and Controlling the Speed in Highways using MEMS Sensor
}

\author{
M.Sathesh M.E ${ }^{1 *}$,J.Shanmugapriyan ${ }^{2}$ \\ ${ }^{1}$ Department of Embedded System Technologies, Vel Tech Multi Tech Dr.Rangarajan \\ Dr.Sakunthala Engineering College, Avadi, Chennai \\ ${ }^{2}$ Assistant Professor, Vel Tech Multi Tech Dr.Rangarajan Dr.Sakunthala Engineering College, Avadi, Chennai \\ *Corresponding Author Email: sathesh1810@gmail.com
}

\begin{abstract}
This Paper describes about the spotting of an over speed of a vehicle in highway and controlling the speed of a vehicle whenever there is an uneven Condition Occurred. And alert the speed offence to control room and toll gate, the devices like Controller, RFID reader, MEMS sensor, GSM\&LCD are used. The System are controlled by a microcontroller, The Micro-Controller plays an important role to do a desired task. The controller obtains the MEMS sensor, which is used to sense speed of the vehicle, Vehicle has RFID tag when speed violation take place where RFID reader reads the tag and identify the vehicle and send the information through GSM module. We design a project in such a manner that it gives an alert signal to the driver if he does not react to that, than two condition occurred first speed is been controlled and other speed offence fine is send control room and toll gate whenever vehicle crossing its maximum speed limit.
\end{abstract}

Keywords: MEMS sensor, LCD, RFID, GSM.

\section{Introduction}

Now a day's as population increases, were are there so much of vehicles in our city were we find a so many of an accidents, especially we find so many accidents in highway because where there is no speed limit were people commit mistake and lose their control on the High way and roads, and accident occurs, and15$20 \%$ of an accident occurs on highway so there is no safety, to secure the person we design a project in simple manner. We Design a project based on MEMS application, collecting the required data from sensors and driver which are attached to microcontroller as well as RFID reader and Monitor each moment at vehicle condition. The vehicle condition status has been updated to a specific mobile phone through GSM .In case a uneven condition occurred then getting alertness through mobile phone and as well as to police station and toll gate. Microcontroller is controls the all devices and performs a specific task. The design of project bring useful for controlling the over speed vehicle in highways, produce alert signal to driver as well as transfer the vehicle information to nearest Police station and toll gate in case of speed violence where speed offence fine will be opposed in toll gate . Due to which there is a less risk of an accident and were police can easily monitor were hit and run or any theft are also been identified easily and were police does not have to struggle were they can easily monitored.

At present there are so many methods to detect the speed where speed of the vehicles are controlled and displayed, where vehicles speed is controlled they are monitored within the zone or range two different units are present transmitter unit and receiver unit when the object (vehicle)comes under the transmitter zone where vehicle speed is been controlled by an receiving signal than speed is decreased and keep the vehicle speed constant until it moved out of an transmitter zone, which is not completely safe and it has some risk when it crosses over the zone there is no speed controlled in that time chances of risk may take place.[1]

The other method over speed detection on highways where system aim is to identify it early and alert of an dangerous vehicle pattern where if the speed increases there will be an alert (buzzer sound) to driver and police but it can be ignored by driver and police cannot able to track the rider in highways it will be a difficult task. [2] .another method to reporting and display of speed violation and it gives an alert if road works, school zone in this laser transmitter sense the load entered by vehicle and receiver part sends to microcontroller, receiver part consists of LDR light dependent resistor if the speed exceeds the information are send to the pc and camera capture the vehicle and send to data base system in this system shortest distance zones are covered, particular zone only will be monitored so there are risk of accident in other zones.[3] The paper is organised as follows: Section II presents Existing method. Section III presents the proposed system. Section IV describes the overall system design. Section V describes the simulation and hardware result. Section VI describes about the future work. Section VII conclusion of the paper.

\section{Existing Method}

In existing system speed detection of an vehicle are done by using radar gun and at which it reads the speed if speed reaches to the maximum level the details of an vehicle is sent to nearest control room, Where in this system consumption of time is more. And the other system to detect the over speed they use RF to control the speed with an distance or within the range where it monitors the speed, keep the vehicle in constant speed within the range of an system, after that it goes as same in this system inaccuracy may occur and after the range over speeding of an vehicle may occur and chance of risk are more. 


\section{Proposed Method}

To overcome the problems on existing system we have introduced this proposed system, where in this system we can able to detect the over speed of an vehicle and control the speed, Mems sensor is used to detect and measure the speed of an vehicle, along with that RFID tag are also present in the system which contains information and details of the vehicle, once the speed is reached to the maximum level RFID reader read the tag and immediately it alerts the driver and if he does not respond to that alert ,within 4-5 seconds automatically speed will be controlled. With the help of GSM module it sends the information details of the vehicle near to highway control room and toll gate so that once they reach toll gate over speed fine will be imposed, due to this there is a less chance of accident in highway and easy to monitor for highway police.

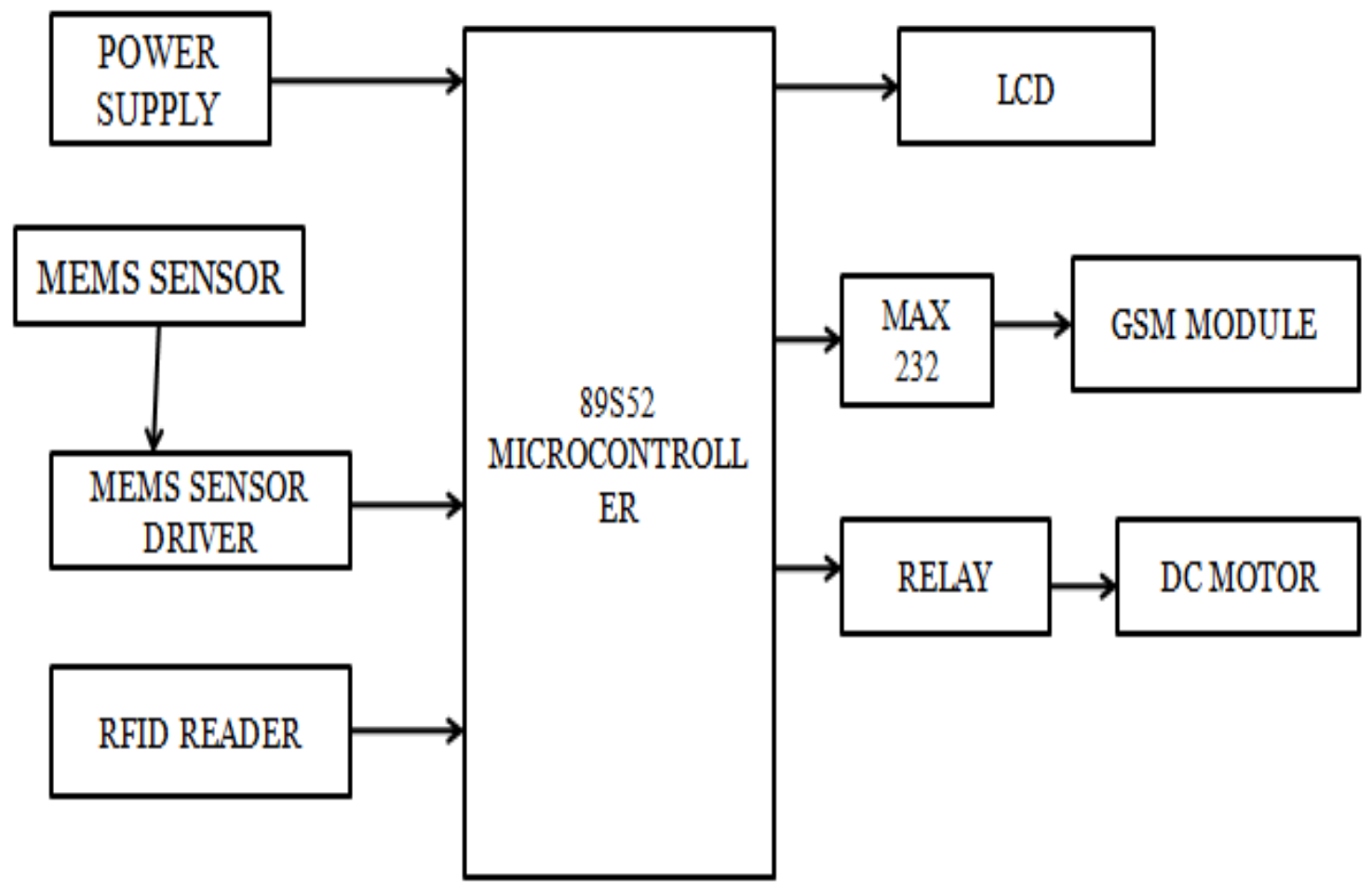

Fig. (a): Overview of a proposed system

\section{Overall System Design}

The system has been designed in such a way that there are three mode of operation that are normal mode, over speed mode and over speed control mode where speed is detected by Mems sensor with the help driver it is connected to microcontroller where it sense the over speed or normal speed than accordingly it performs the task with help of a relay we are able to switch the mode.

when vehicle reach maximum level speed where we assume motor as a vehicle that when over speed mode is activated it performs a task accordingly where in LCD display over speed detection will be shown and there is an alert and buzzer to aware the driver is crossings its speed if he does not respond to that with an seconds the speed will be controlled and then over speed control mode is activated (that motor gets to the normal mode) and RFID tags gets activated and once RFID reader reads the tag which consist of vehicles details and information and with the help of GSM module information is send to highway control unit and toll gate .In figure (b) shows the overview of a hardware design.

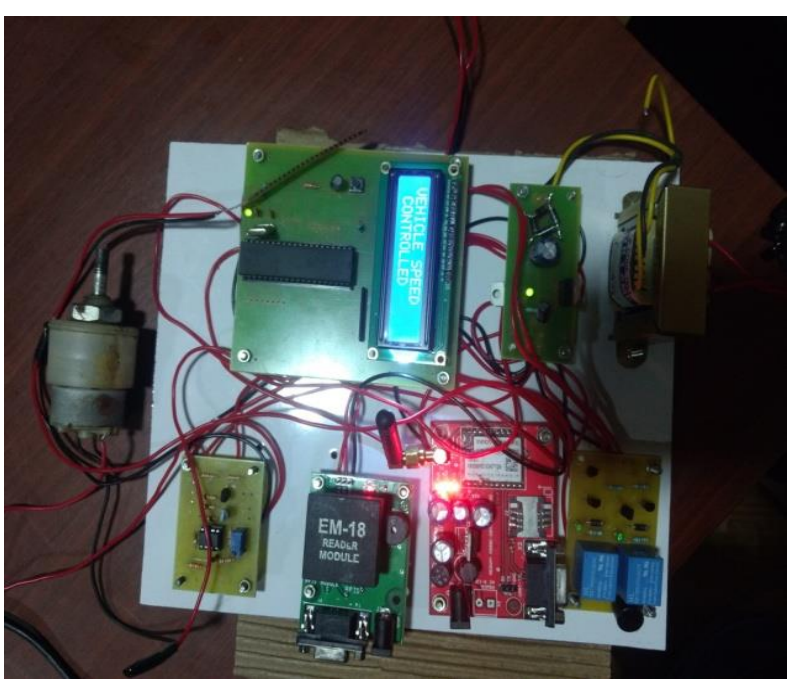

Fig. (b): Overview of hardware design

Where it shows the hardware setup and arrangement of the unit And in Figure (c) describes about the circuit that are designed for over speed detection using Mems sensor and RFID reader and LCD. 


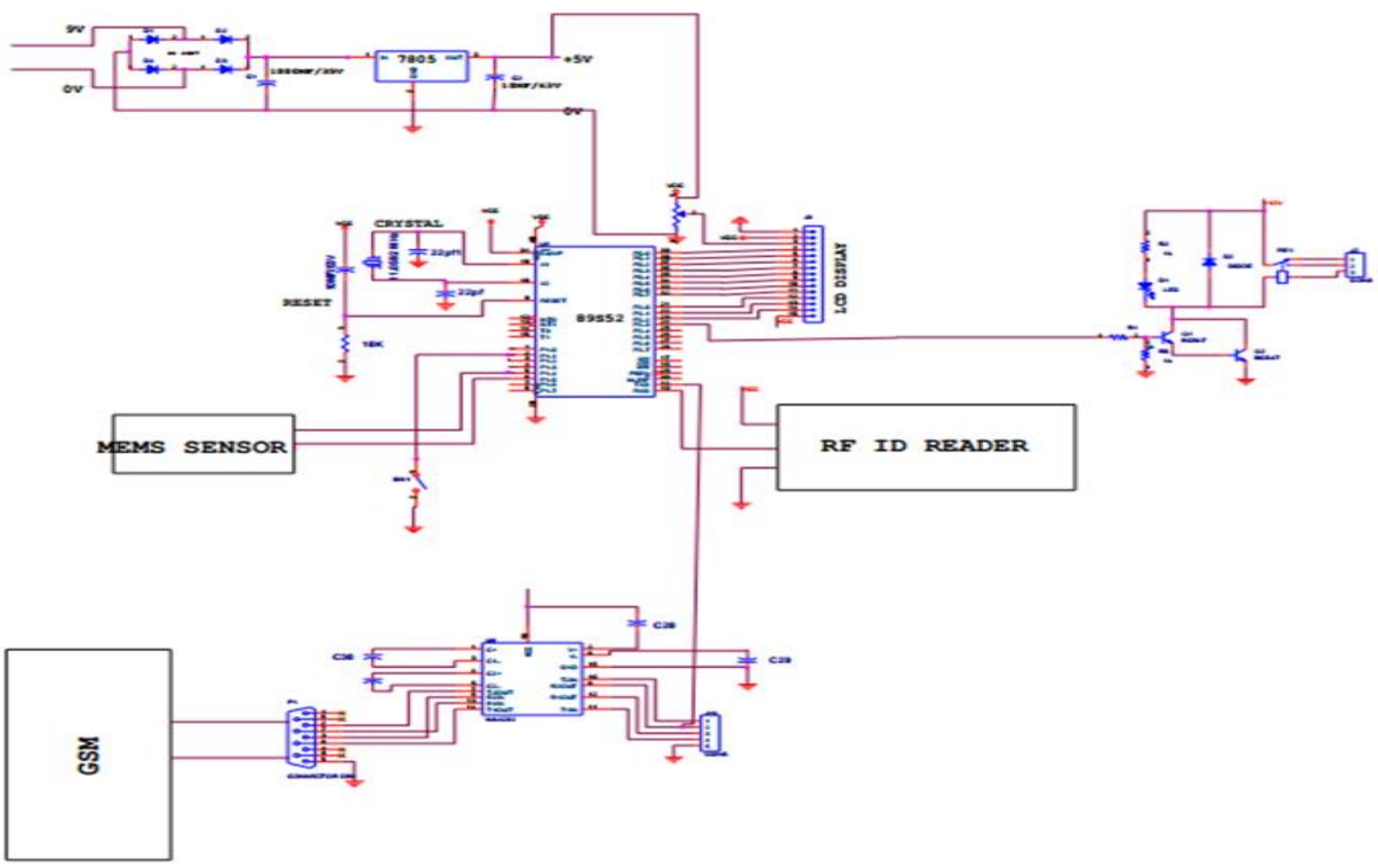

Fig. (c): Circuit diagram of a proposed system

\section{Result}

In this section we discuss about the simulation and hardware result. The simulation design was developed using PROTEUS software. The coding is done using embedded c language where in this we have two modes one is normal mode and over speed control mode Figure(c) shows the simulation result for normal speed mode when speed is normal the blue LED will glow to indicate that speed is normal and task performs accordingly.

In Figure (d) the simulation result of an over speed when it reaches to maximum speed where all led will glow and indicate the over speed.

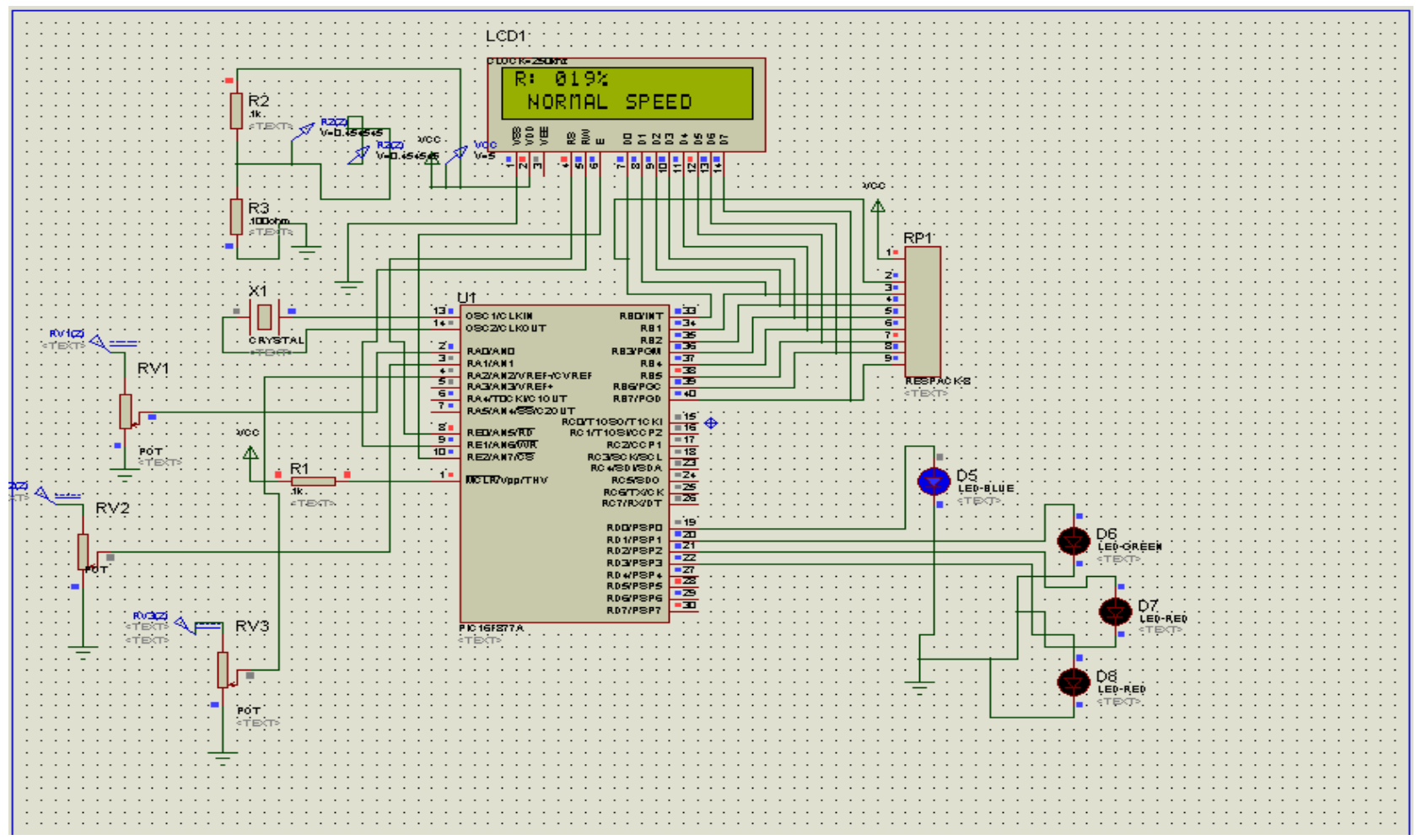

Fig. (d): Simulation result for an normal mode condition 


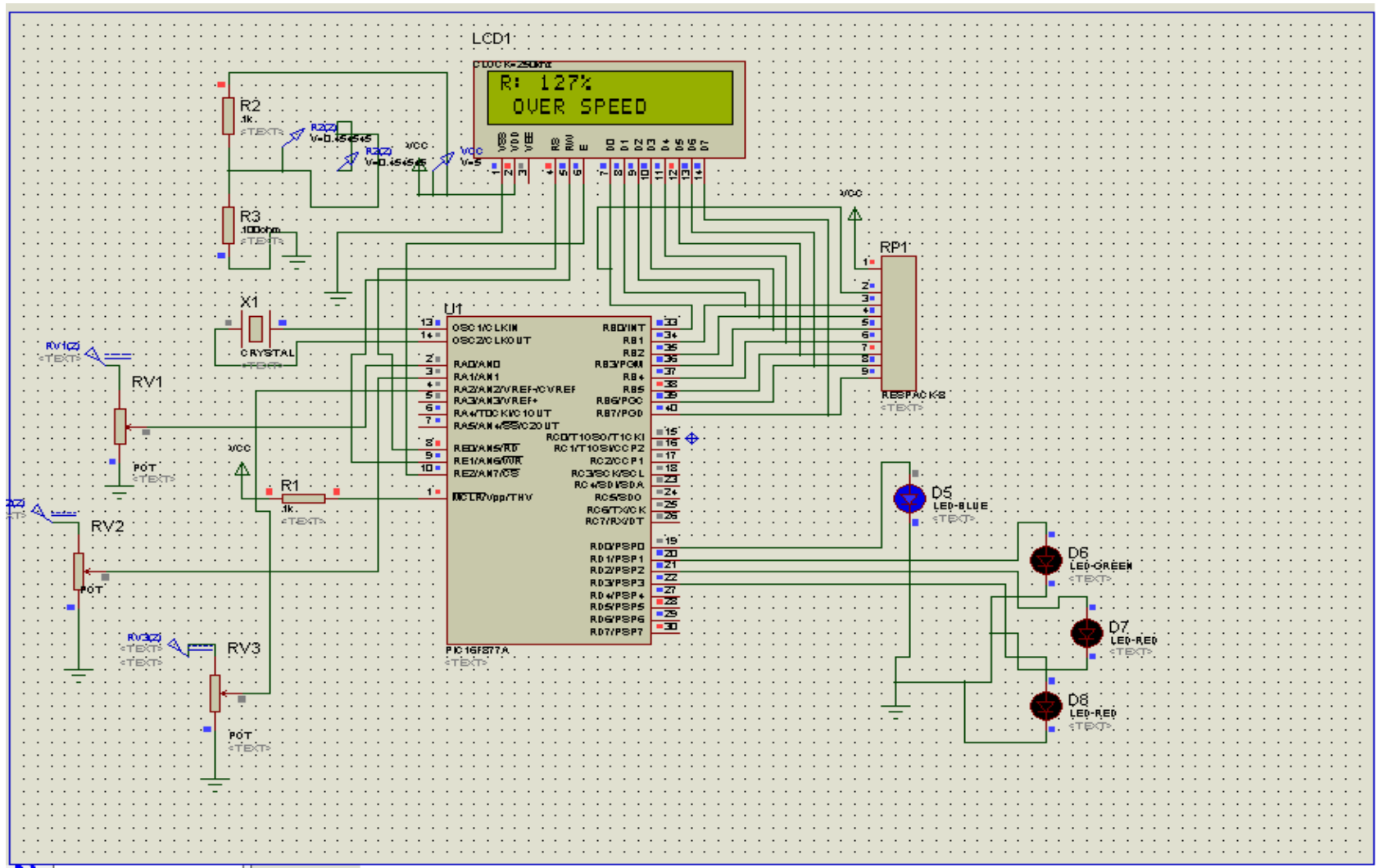

Fig. (e): Simulation result for an over speed mode

Hardware result in this we will able to see the different mode of an system and due to Mems sensor are able to detect the speed where once power supply is given the motor runs which is assumed as vehicle and when the motor run slower, Than normal mode is activated. As shown in figure (f).

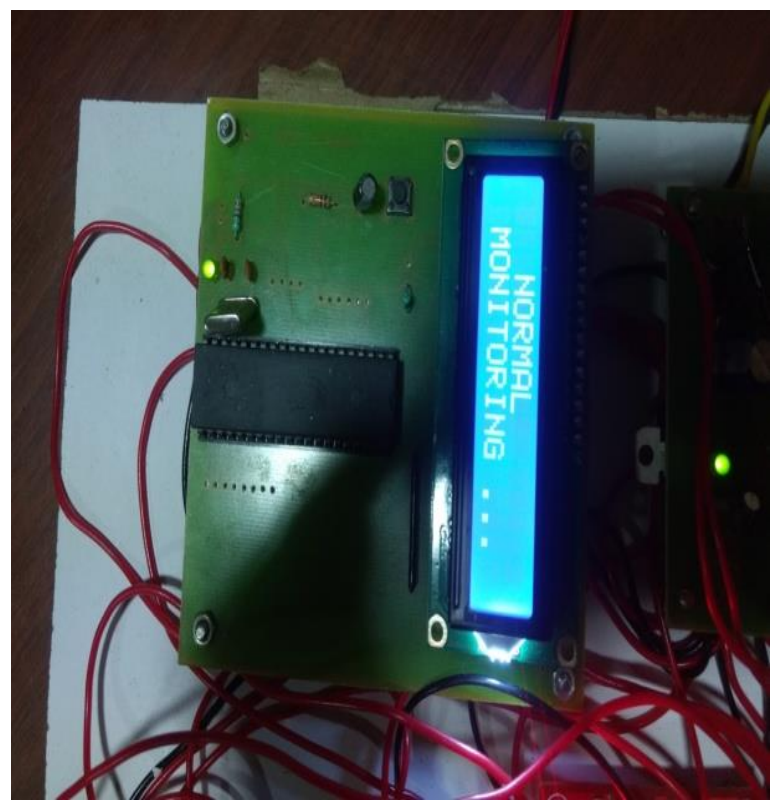

Fig. (f): Normal monitoring mode

When Mems sensor and driver sense that the vehicle speed is in normal modes that are detected or monitored are shown in LCD display figure (f).

Once motor reaches to maximum speed (where we assume that motor as a vehicle), when it exceeds the limit with the help of relay where going to control the motor with a seconds if the driver does not respond to the alert, over speed and control mode get activated and speed is controlled and simultaneously sms and data of that particular vehicle are also sent which is stored in RFID tag that is been read by RFID reader and with the help of an GSM module sms is carried out to nearest control room and toll gate as shown in figure $(\mathrm{g})$.

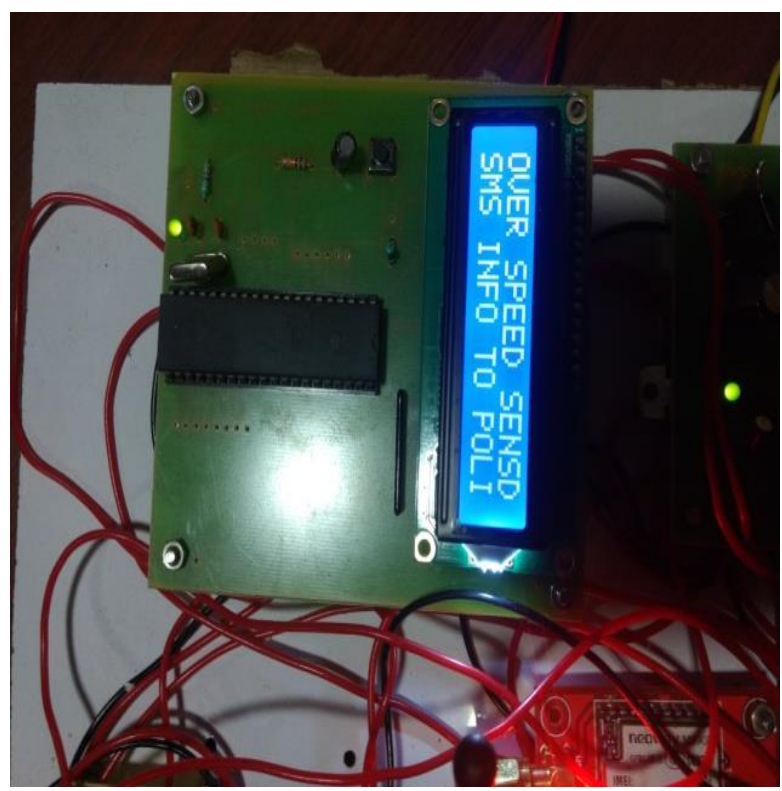

Fig. (g): Over speed control mode

As shown in figure (h) with the help of GSM module SMS are sent with the time and display the detailed information of an identified vehicle has crossed its speed limit. 


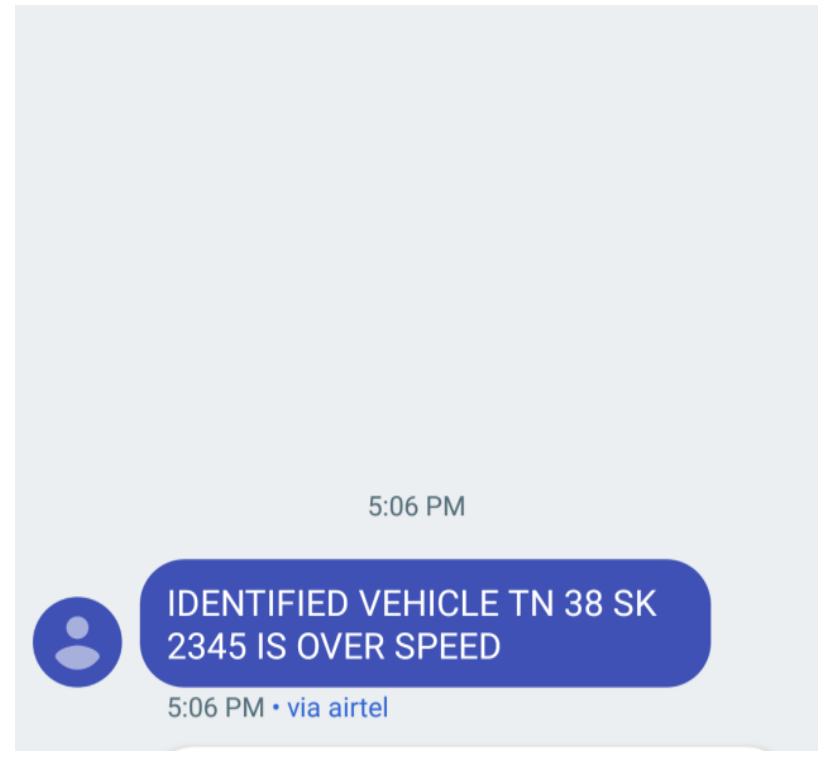

Figure (h) Details of an over speed of a vehicle is send to control room

\section{Future Work}

In this proposed system where we are able to spot the accurate speed with the help of Mems sensor and its driver once it reaches to the maximum speed it gives alert to driver as well sms is sent to police control room and toll gate within seconds speed are also controlled and monitored for normal mode but we are using RFID tag to send details late in future we can use camera for capturing of image of an number plate and easily identification of driver by face recognisation and multi angle position to be captured in order to get more accurate vehicles and hit and run also will be caught easier.

\section{Conclusion}

Major accident all occurred in highways it can be controlled by this system we can able to reduce risk of an accident, we have an automatic speed reduction of an vehicle, When vehicle crosses its maximum speed the relay gets activated automatically and reduces the speed of the Motor. Speeds of the vehicles are controlled and normal mode speed is been monitored then there is a less risk of an accident in highways. And police will able to identify the vehicle easily and they can oppose penalty for a concerned vehicle.

\section{References}

[1] Vishal Pande, Malhar Mohite, Supriya Mhatre, Siddhesh Desai, Anjali Kumari, International Journal of Advanced Research in Electrical, Electronics and Instrumentation EngineeringVol. 4, Issue 4, April 2015 Autonomous Speed Control of Over Speeding Vehicles Using Radio Frequency

[2] Monika Jain, Praveen Kumar, Priya Singh, Chhavi Narayan Arora, Ankita Sharma, International Journal of Computer Science and Mobile Computing A Monthly Journal of Computer Science and Information Technology, Vol. 4, Issue. 4, April 2015a system Detection of over Speeding Vehicles on Highways

[3] Ni Hlaing, Zaw Min Min Htun, Hla Myo Tun International Journal Of Scientific \& Technology Research Volume 4, Issue 07, July 2015 Design And Implementation Of Pc Based Over Speed Violation Management For Vehicles On Highway

[4] Amarnarayan, Challa Saikumar, Chandra Mohan, Ajaykumar, Sridhar International Journal of Combined Research \& Development (IJCRD) May 2016 Automatic Over speed Controlling of Vehicle
[5] M. Saritha Reddy, K.Swetha, A.Yamini, K.Ravi Teja International Journal of Advanced Research in Electrical, Electronics and Instrumentation EngineeringVol. 6, Issue 4, April 2017Zigbee Based Speed Sensing System andProviding Alarm of Over Speed

[6] Sarmad Majeed Malik, Muhammad Asad Iqbal , Zohaib Hassan, Tauseef Tauqeer, Rehan Hafiz Research gateConference Paper . September 2014Automated Over Speeding Detection and Reporting System

[7] Syed Ahson Mohammad Ilyas Edited a RFID handbook, Applications, Technology, Security, and Privacy.

[8] Elena Gaura and Robert M. Newman published a book on 2006 based on the Smart MEMS and Sensor Systems.

[9] S.V.Manikanthan and K.Baskaran "Low Cost VLSI Design Implementation of Sorting Network for ACSFD in Wireless Sensor Network", CiiT International Journal of Programmable Device Circuits and Systems,Print: ISSN 0974 - 973X \& Online: ISSN 0974 - 9624, Issue : November 2011, PDCS112011008.

[10] T. Padmapriya and V.Saminadan, "Handoff Decision for Multi-user Multiclass Traffic in MIMO-LTE-A Networks", 2nd International Conference on Intelligent Computing, Communication \& Convergence (ICCC-2016) - Elsevier - Procedia of Computer Science, vol. 92, pp: 410-417, August 2016.

[11] S.V. Manikanthan , T. Padmapriya "An enhanced distributed evolved node-b architecture in 5G tele-communications network" International Journal of Engineering \& Technology (UAE), Vol 7 Issues No (2.8) (2018) 248-254.March2018. 